\title{
Increase of stability of underground works
}

\author{
K. Weiglová ${ }^{1}$ \& P. P. Procházka ${ }^{2}$ \\ ${ }^{1}$ VUT in Brno, Institute of Geomechanics, Brno, Czech Republic \\ ${ }^{2}$ CTU in Prague, Civil Engineering, Prague, Czech Republic
}

\begin{abstract}
In this paper the influence of additional structural precaution is studied for an exceptional tunnel facing in extraordinarily difficult conditions. In the case considered here artificial roofing is created by a system of horizontally drilled reinforced piles, which are prepared before drilling current purchase in the frame of tunnel engineering. They are positioned at three height levels above the expected vault (calotte) of the future tunnel, but in the model only one level is assumed (the others are built up for the sake of safety). The influence of the roofing has been verified on two independent models (with and without roofing), treated both experimentally and numerically.

Both physical scale models (experimental) and the numerical approach confirmed extremely high improvement of long-lasting stability of the underground work and settlement of the overburden. Using coupled modeling (experimental and numerical) enabled one to quantify the values of the necessary parameters. In numerical models the influence of pile roofing was measured in deformation and stress states of the rock mass. From the results obtained during numerical experiments with different types of roofing the deformation of the overburden was basically restricted. Depending on the material, the deformation dropped by as much as 95 percent in comparison to the non-reinforced case.

In the paper the approach consisting of couple modeling - experimental and numerical - will be described. The experiments are prepared on scale models in stands (basins with glazed front sides and the length of 2-6 m), where physically equivalent materials simulate the real situation. Based on similarity rules very good agreement with reality is attained.
\end{abstract}

Keywords: scale modeling, stability of underground works, tunnel face, coupled modeling, artificial roofing. 


\section{Introduction}

Coupled and comparative models consist of connection of results from mathematical and experimental models. In the experiments physically equivalent materials are used, which simulate real states in reality. In order to get the real situation in the scale model, similarity rules have to be fulfilled, $[1,2]$. In this way the experimental model becomes real. The structure of tunnel behavior in extreme conditions is studied in this paper. The tunnel is built up in the city of Brno, Czech Republic, and the conditions which should be taken into consideration require involvement of predisposed cracks, which appear in the neighborhood of the tunnel lining. In the mathematical model the eigenparameter technique is exploited [3]. This approach is generalized starting with nonlinear behavior of the rock, which is described by Mises hypothesis [4]. Previously [5], such a combination of eigenparameters and starting plasticity state was applied. For details see [6], where coupled modeling based on mutual affection of scale experimental models and numerical models based on the generalized eigenparameter technique is described.

Here the influence of predisposed cracks on tunnel stability is studied. In this sense instead of eigenparameters the coefficient of internal friction $\varphi$ and the cohesion (shear strength) $c$ are considered as free parameters in the coupled modeling. The basic idea of the former approach is still considered, i.e. influence matrices are first created for the fully elastic case and a linear hull of plastic, eigenparameter and elastic stages are considered in such a way that the measured values in experimental models and that of numerical models at selected points are as close as possible. For this reason, unit internal parameters (the coefficient of internal friction) and shear strength are introduced. As the influences of the latter parameters are solved in elastic (linear) medium, influence matrices are easy to construct. The auxiliary functional of variances of differences in stresses or displacements is formulated and minimized for unknown values of the design parameters $\varphi$ and $c$. Once they are determined the critical situation can be assessed, which covers visible damage of the structure, strain observation and possible limitation, and, eventually, failure of the structure.

\section{Description of the situation on site}

In the city of Brno, Czech Republic, Tunnels Dobrovského (Street) of the Great City Circuit will be an important component of the communication system of the city as well as of the road network of the Czech Republic and of an international network. The tunnels are driven in the length of about $1000 \mathrm{~m}$. The tunnels are engineered in parallel with the axial distance of about $80 \mathrm{~m}$. The thickest complex with the largest area of Neogene sediments in Brno and surroundings is chalky clay, often with a perceptible disintegration, so-called fissured texture fine grained soil with very high plasticity. Volume instability is expected in this are of construction. In Fig. 1 the longitudinal cross-section is seen with various layers of geological positions. 


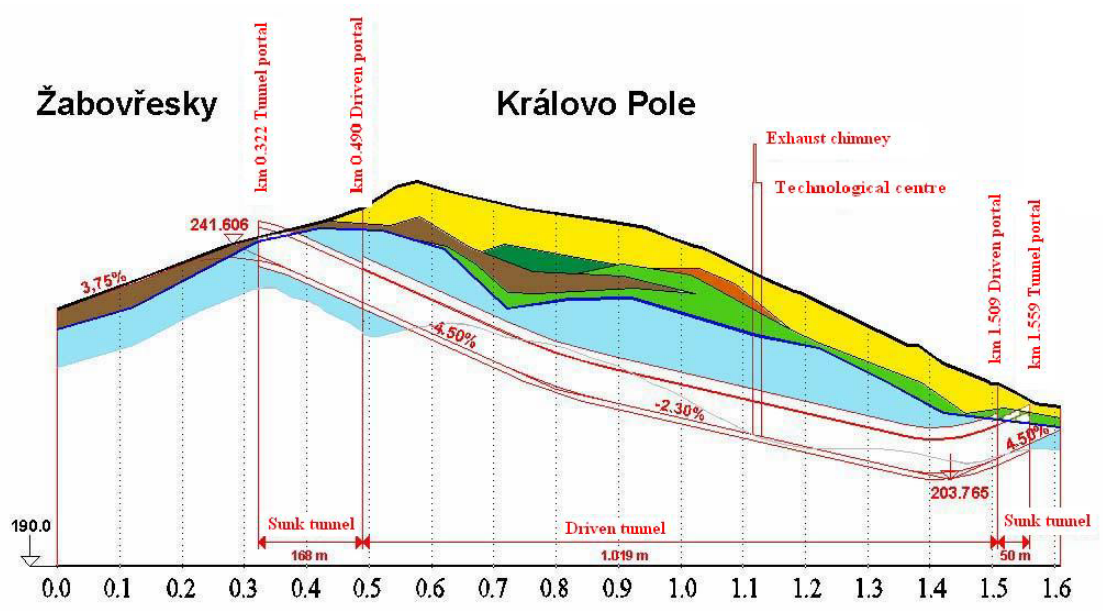

Figure 1: Longitudinal cross-section of the tunnel.

Calcareous clay containing $10-30 \%$ of carbonate minerals are the prevailing minerals on site. An admixture of classic grains (quartz, muscovite, feldspars) is contained in the rock, as well as the calcareous to siliceous shells of microfossils. Pyrite and organic substances, clayey minerals in the amount of 50-60\% and the presence of smectites of the illite-chlorite type also state the essential properties of the rock.

Unlayered or inconspicuously layered sediment sherd-like break-off to shelly zones with the fissured texture surfaces of discontinuities of different directions are observed with piece-like slopes disintegration in zones of weathering pelitic microstructure granulation of $0.002-0.0005 \mathrm{~mm}$. From the point of view of contraction and swelling the Brno clay is one of the most problematic soils. The reason is the presence of smectites Neogene clay Brno-Královo Pole: high values of the plasticity index, high values of the activity index (2.2) and the cofactor of volume swelling $\mathrm{B}=7 \%$, sensitivity $\mathrm{St}=1.3$ (at rigid consistency) - which results in development of swelling pressures.

In Fig. 2 results from triaxial tests are depicted showing basic mechanical properties of the material.

\section{Experimental}

The scale models are prepared to deliver data for accurate numerical modeling of problems required in geotechnical engineering. Back analysis or coupled modeling is one of the possible approaches to identify deformation of tunnel lining, tunnel surrounding rock, crack occurrence and finally, identification of failure of the structure. In our case of extremely exacting conditions (shallow foundation of the tunnels, clayey soil, loose rock, etc.) extreme attention has to be drawn to other certain special items: 
- Establishing the stability of the provisional rock pillar (middle pillar) between the galleries when driving the tunnel by vertical articulation of the stope including the determination of the optimum length of the attack.

- Examination of the rate of pushing up the footwall into the floor up to the failure of the provisional rock pillar.

- Whole tunnel profile (section), considering the effect of the lining, the system was loaded up to the limiting state of failure with formation of shear surfaces.

To maintain the relation between the model and the reality, it was necessary to fulfill the following conditions:

- The models must be geometrically similar to reality, see the following text

- Actions taking place in the model and in the work must belong to the same class of actions

- The beginning and marginal conditions in the model, which are expressed in the dimensionless model, must be numerically identical with the dimensionless conditions in the work.

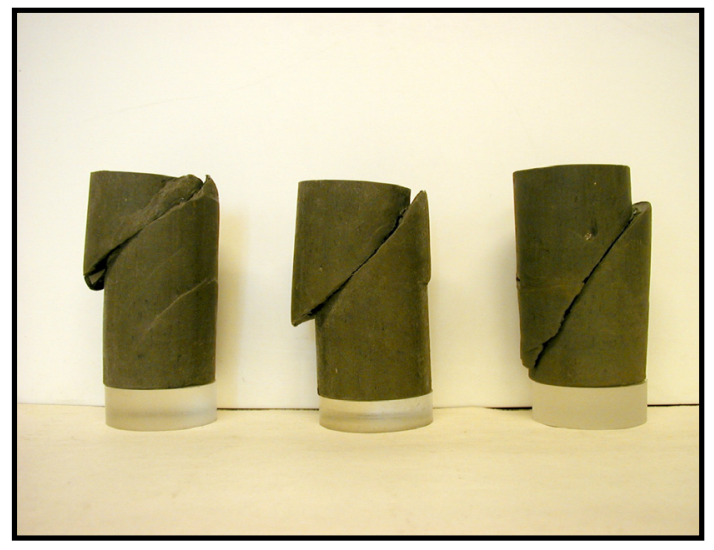

Figure 2: Samples of Brno clay after the test in the triaxial apparatus.

The dimensionless arguments of the same nature must be numerically identical in the model and in the work.

Models of identical materials are built up on the basis of dimensional analysis and in accord with the theory of similarity, which make them possible to be feasible to the preliminary qualitative and theoretical analysis of problems and, at the same time, to the selection of the system determining dimensionless magnitudes.

In physical models several similarities have to be obeyed. One of the most important is geometrical similarity; it is proportionality of dimensions and identity of angles between model and modeled object in the whole range of the model. Altogether, possibility and technique of bringing of forces, time factor and other aspects, technical possibilities, assign linear scale of the model are objective of the modeled geotechnical problem. It is the aspect ratio, which plays essential role, in which length dimensions of the model are reduced against the reality. 
If $1 / \alpha_{1}$ is the length scale, i.e. the ratio of lengths on model and reality and a is the ratio of bulk densities

then it can be defined for

$$
\mathrm{a}=\rho_{\text {model }} / \rho_{\text {reality }},
$$

$$
\begin{array}{ll}
\text { forces } & \mathrm{P}_{\text {model }}=\mathrm{a} \cdot\left(1 / \alpha_{1}\right)^{3} \cdot \mathrm{P}_{\text {reality }} \\
\text { stresses } & \sigma_{\text {model }}=\mathrm{a} \cdot\left(1 / \alpha_{1}\right) \cdot \sigma_{\text {reality }} \\
\text { deformations } & \varepsilon_{\text {model }}=\text { a }\left(1 / \alpha_{1}\right) \cdot \varepsilon_{\text {reality }} .
\end{array}
$$

To simulate processes taking place in the rock material in the most perfect way, rock environment is replaced in the model from equivalent materials by their determinate physical and mechanical properties according to the model laws. The scale of model agrees with the rock properties and respects the character of failures simulating those in rock material. The models are constructed from mixture of various, mostly easy available materials: On the basis of an extensive set of laboratory experiments (ballotine, bentonite, glycerine, sand, graphite and ferrosilicium) physically equivalent materials were selected as:

- $\quad$ sand with an admixture of fat A00 $(99.5 \%+0.5 \%)$

- ballotine with an admixture of fat A00 $(99 \%+1 \%)$

- ferrosilicium with an admixture of fat A00 $(99.5 \%+0.5 \%)$

The scale of the model is chosen according to the smallest part of the structure (in our case the thickness of the outfit), which must be feasible.

In Fig. 3 face view of the stand with the structure illustrate the model geometry. The reinforced basin (the stand) with glazed front wall shows possibilities of observation of changes in models (or, equivalently, in reality) not only from the point of view of quantity but also from the standpoint of quality, as time dependent processes can be qualified.

In Fig. 4 detailed view of artificial roofing and future tunnel are depicted. The experimental models in stands simulate the real situation on a scale $1: 20$. In the upper part of the picture the horizontal piles are seen, which are created from rebar reinforced concrete. They can be in 3D model considered as beams with given bending stiffness.

In Fig. 5 detailed view of the tunnel in the scale model (the stand) is shown with measurement equipments. Dilatometers are positioned inside of the tunnel hole and on the face of the lining also tensometer gauges are stuck.

\section{Numerical approach}

The aim of this paragraph is to show some possible relations involving stresses (or strains) and eigenstresses (or eigenstrains) in composite structures. Consider the domain $\Omega \in R^{3}$ with boundary $\Gamma$, describing the shape of the body under study. Inside this body n subdomains are situated $\Omega_{1}, \Omega_{2}, \ldots, \Omega_{n}$. The boundary $\Gamma$ is split as follows: $\Gamma \equiv \Gamma_{u} \cup \Gamma_{p}, \Gamma_{u} \cap \Gamma_{p}=0$.

On $\Gamma_{u}$ the displacements $u_{i}=\bar{u}_{i}, i=1,2,3$ are prescribed, while on $\Gamma_{p}$ the tractions $p_{i}=\bar{p}_{i}, i=1,2,3$ are given. The primed quantities are given. 


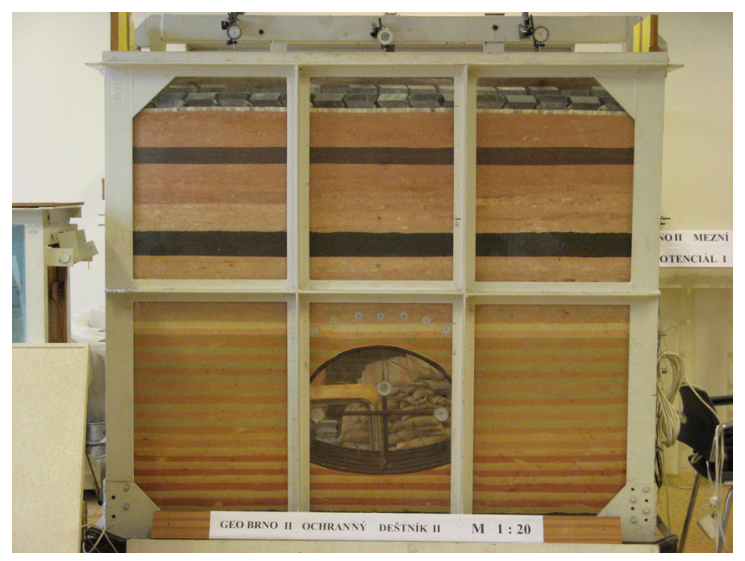

Figure 3: View of the stand.

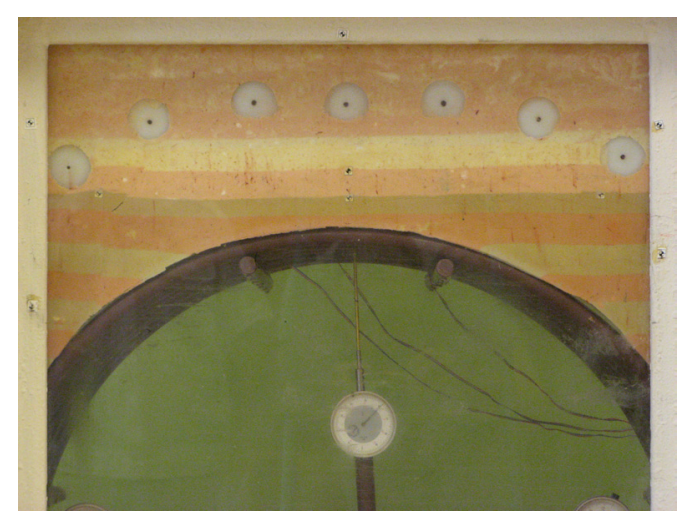

Figure 4: Detailed view of the stand with artificial roofing.

Let us consider that there is negligible plastic behavior in lining (being mostly compressed). In this case Hook's law with material stiffness $\boldsymbol{L}$ holds

$$
\sigma_{i j}=L_{i j k l} \varepsilon_{k l}+\lambda_{i j}, \quad \lambda_{i j}=-L_{i j k l} \mu_{k l} \quad \text { in } \Omega
$$

The real displacements $\boldsymbol{u}$, strains $\boldsymbol{\varepsilon}$, and stresses $\sigma$ are unknown in the latter relation, so are the eigenstrains $\boldsymbol{\mu}$.

The symmetric polarization stress tensor $\tau$ is introduced as:

$$
\sigma_{i j}=L_{i j k l}^{\text {lining }} \varepsilon_{k l}+\tau_{i j}
$$

Since both $\sigma$ is statically admissible, it holds:

$$
\begin{gathered}
\frac{\partial \sigma_{i j}}{\partial x_{j}}=\frac{\partial\left(L_{i j k l}^{\operatorname{lining}} \varepsilon_{k l}+\tau_{i j}\right)}{\partial x_{j}}=0 \text { in } \Omega \\
\tau_{i j}-[L]_{i j k l} \varepsilon_{k l}-\lambda_{i j}=0 \text { in } \Omega
\end{gathered}
$$




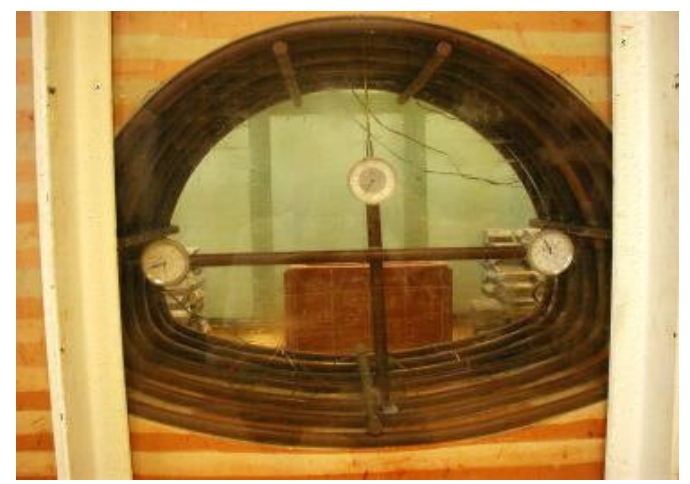

Figure 5: Detail of the tunnel with reinforcement and measuring equipments.

and

$$
[L]_{i j k l}=L_{i j k l}-L_{i j k l}^{\text {lining }} .
$$

Note that the relation (3) can be written in more details as:

$$
\sigma_{i j}=2 G^{\text {lining }} \varepsilon_{i j}+\delta_{i j} \frac{2 G^{\text {lining }} v^{\text {lining }}}{1-2 v^{\text {lining }}} \varepsilon_{k k}+\tau_{i j}=2 G^{\text {lining }} \varepsilon_{i j}+\lambda^{\text {lining }} \delta_{i j} \varepsilon_{k k}+\tau_{i j}
$$

and Kronecker's delta $\delta_{i j}=1$ for $i=j$ and it is equal to zero otherwise. Material constants $G$ and $\lambda$ are Lame's constants ( $G$ is also referred to as the shear modulus), $v$ is the Poisson's number.

Applying the Green theorem to (4) after multiplying each $i$-th equation by $\varphi_{i}$, which is not identically zero in $\Omega$ and integrating over the domain yields

$$
-\int_{\Omega}\left(L_{i j k l}^{\text {lining }} \varepsilon_{k l}+\tau_{i j}\right) \frac{\partial \varphi_{i}}{\partial x_{j}} \mathrm{~d} \Omega+\int_{\Gamma}\left(L_{i j k l}^{\text {lining }} \varepsilon_{k l}+\tau_{i j}\right) \varphi_{i} n_{j} \mathrm{~d} \Gamma=0
$$

After decoding the latter equations in the sense of finite element method it is seen that the nonlinear behavior in the lining is suppressed and only rock has to be involved into plastic computation. Hence, using the back analysis (or coupled modeling) of experimental and numerical mutual influence, identification of eigenparameters are obtained for the rock without pile reinforcement. Using the material behavior from this state and extend it to the reinforced case, necessary length of the piles can be computed from the mathematical model. It is worth noting that parametric study on this problem of the necessary length of horizontal pile beam can hardly be conducted in experiment in the whole extent. On the other hand, numerical study is very cheap, not only from the standpoint of computation, but also because of very large extent of simulations of material and structural combinations.

\section{Example}

The numerical problem starts with the solution of plastic stage obeying generalized Mohr-Coulomb hypothesis with the following material parameters: 
Modulus of elasticity $E=1000 \mathrm{MPa}$

Plastic $E=800 \mathrm{MPa}$

Residual $E=500 \mathrm{MPa}$

Poisson's ratio $v=0.25$

Plastic equals residual $v=0.46$

Shear strength $C=0.08 \mathrm{MPa}$

Plastic $C=0.06 \mathrm{MPa}$

Residual $C=0.02 \mathrm{MPa}$

$\tan \varphi=24^{0}$

Plastic $\tan \varphi=30^{\circ}$

Residual $\tan \varphi=10^{\circ}$

Volume weight $\rho=2100 \mathrm{~kg} / \mathrm{m}^{3}$.

Using the above said parameters describing material properties, which correspond with the class $\mathrm{R}_{3}$ of the rock according to geological standards. The tunnel lining is made from concrete and no plastic behavior is assumed. After computing the plastic state with the above parameters, initial stage is created and the eigenstrains can be introduced.

The measured values were also vertical deflections on the contact of lining and rock. The values were taken from a scale model built up in stands. There were nine measurement points along the lining; the values at symmetric points were averaged.

In Fig. 6a) hypsography of vertical stresses in the domain without reinforcement is depicted and in Fig. 6b) hypsography of vertical stresses in final stage after optimization of the piles is displayed. Difference between these two pictures is not as much distinct as supposed.

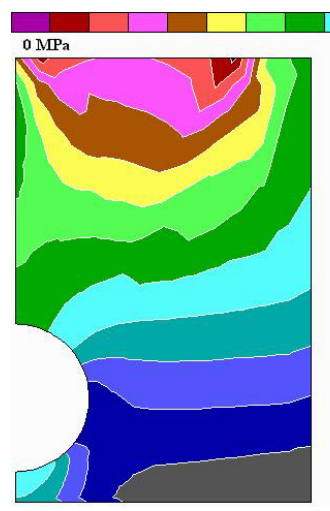

a)

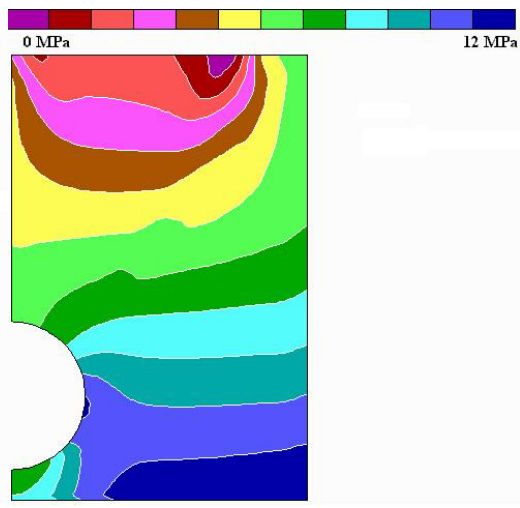

b)

Figure 6: Vertical stresses in a) plastic stage and b) after reinforcement.

In Fig. 7 relation between the length of pile beams and the length of purchase during tunneling is shown. The pile beams are designed according to classical beam's theory. 


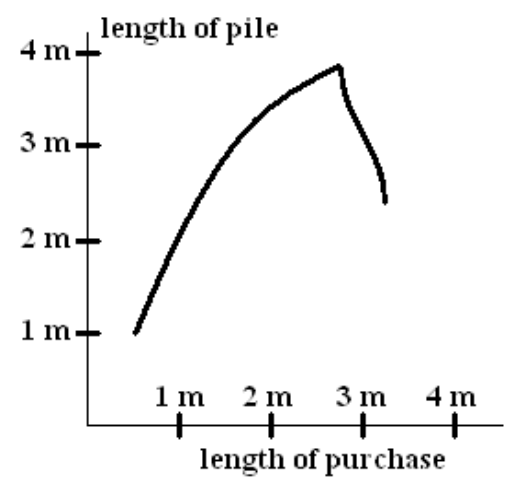

Figure 7: $\quad$ Length of pile necessary for stable engineering.

\section{Conclusions}

In this paper coupled modeling helps to assess tunnel face stability from the viewpoint of auxiliary reinforcement above the future tunnel (roofing). Scale models and numerical treatment lead to very close view into the situation on future site. The model, of course, verified and confirmed the right decision to build up roofing from the concrete reinforced piles. But not only this: they enabled us to determine necessary length of piles (considered as a beam in the 3D finite element model) to ensure the stability of engineering during tunneling. This necessity is induced by the fact that the reinforcing piles can be set up only during the process of construction of tunnels.

\section{Acknowledgement}

Work on this project has been financially supported by GACR, project No. $103 / 06 / 1124$. Sponsorship of CIDEAS is also acknowledged.

\section{References}

[1] Kožešník, J. (1983). Theory of similarity and modelling. Academia, Prague, 1983.

[2] Head, K. H. (1992). Manual of soil laboratory testing. Wiley, New York, 1992.

[3] Dvorak, G.J. (1992). Transformation field analysis of inelastic composite materials. Proc. R. Soc. London A 437, 1992, 311-327.

[4] Duvant, J. and Lions, J.P. (1972). Variational inequalities in mechanics. DUNOD, Paris

[5] Procházka, P. and Weiglová, K. (2002). Coupled modelling using DSC and TFA models. Proceedings of the ICTACEM, Kharagpur, 2002, paper No. 105.

[6] Procházka, P. and Trčková, J. (to appear in Solids and Structures). Assessment and Control of Tunnel Structures based on coupled modelling. 